OPEN ACCESS

Edited by:

Preston E. Garraghty, Indiana University Bloomington,

United States

Reviewed by:

Ines R. Violante,

University of Surrey, United Kingdom Fernando Falkenburger Melleu, University of São Paulo, Brazil

${ }^{*}$ Correspondence: Mohammed Z. Allouh m_allouh@uaeu.ac.ae

Received: 16 January 2020 Accepted: 07 May 2020

Published: 23 June 2020

Citation:

Allouh MZ, Al Barbarawi MM Ali HA, Mustafa AG and Alomari SO (2020) Morphometric Analysis of the Corpus Callosum According to Age and Sex in Middle Eastern Arabs: Racial Comparisons and Clinical Correlations to Autism Spectrum Disorder.

Front. Syst. Neurosci. 14:30 doi: $10.3389 /$ fnsys.2020.00030

\section{Morphometric Analysis of the Corpus Callosum According to Age and Sex in Middle Eastern Arabs: Racial Comparisons and Clinical Correlations to Autism Spectrum Disorder}

\author{
Mohammed Z. Allouh ${ }^{1,2 *}$, Mohammed M. Al Barbarawi ${ }^{3}$, Heba A. Ali ${ }^{2}$, \\ Ayman G. Mustafa ${ }^{4}$ and Safwan O. Alomari3,5 \\ ${ }^{1}$ Department of Anatomy, College of Medicine and Health Sciences, United Arab Emirates University, Al Ain, United Arab \\ Emirates, ${ }^{2}$ Department of Anatomy, Faculty of Medicine, Jordan University of Science and Technology, Irbid, Jordan, \\ ${ }^{3}$ Division of Neurosurgery, Department of Neurosciences, Faculty of Medicine, Jordan University of Science and Technology, \\ Irbid, Jordan, ${ }^{4}$ Basic Medical Science Department, College of Medicine, QU Health, Qatar University, Doha, Qatar, ${ }^{5}$ Division \\ of Neurosurgery, Department of Surgery, American University of Beirut Medical Center, Beirut, Lebanon
}

This study sought to examine the influence of age and sex on morphometric measurements of the corpus callosum (CC) within Middle Eastern Arab population, in order to obtain reference data and conduct racial comparisons with previously reported measurements from other ethnicities. Furthermore, it aimed to investigate CC variations that may occur in children with autism. To this end, magnetic resonance images of normal brains were acquired from three different age groups, consisting of children, younger adults, and older adults. Brain images were also acquired from boys with autism spectrum disorder (ASD). The CC length, area, and thickness were measured. The CC length was smaller in children than in the other age groups, but no difference in CC length was found between younger and older adults. The CC area and thickness were greater in younger adults than in children and older adults, and greater in older adults than in children. With regard to sexual dimorphism, the CC area and forebrain volume were larger in male children than in female children. No sex-related differences in CC area or thickness were found in adults. However, the ratio of CC area to the forebrain volume was greater in adult females than in males, owing to the smaller forebrain volume in females. The absolute length of the $\mathrm{CC}$ was greater in older adult males than in their female counterparts. In addition, significant differences in CC measurements were found in comparison to measurements obtained from other ethnicities. Lastly, significant reductions in CC area and thickness were found in boys with ASD compared to their neurotypical peers. In conclusion, age and sex significantly influence morphometric measurements of CC in Middle Eastern Arab population. This study points to the presence of racial differences in CC size. Finally, it reveals that children with ASD display a distinct reduction in CC size compared to neurotypical children of the same ethnicity.

Keywords: autism, corpus callosum, ethnicity, forebrain, magnetic resonance imaging, Middle Eastern Arab, morphometric measurements 


\section{INTRODUCTION}

The corpus callosum (CC) is the largest bundle of commissural fibers in the human brain. It consists of at least 200-300 million fibers that connect the right and left cerebral hemispheres together (Huang et al., 2005; Sakai et al., 2017). This white matter structure plays important roles in transferring sensory, motor, and cognitive information between the right and left cerebral hemispheres (LaMantia and Rakic, 1990). Most of these fibers provide homotopic connections between mirror-imaged areas in the cerebral hemispheres (Huang et al., 2005). However, heterotopic fibers that connect anatomically and functionally different regions of the cerebral cortex in an asymmetric manner are also present (Mooshagian, 2008).

Anatomically, the CC is divided into four distinct regions, consisting of the rostrum, genu, body, and splenium (Sakai et al., 2017). The genu is the most anterior region, near the frontal lobe, while the splenium is the most posterior area, near the occipital lobe. The rostrum is the inferior backward extension from the genu, and the body is the largest area of the CC, located between the genu and splenium (Jones, 1985).

Several studies had previously investigated whether there are any variations in the CC that could be attributed to age or sex. Weis et al. (1993) reported significant age-related changes in the anterior parts of the CC (genu and anterior segment of the body) and concluded that the total area of the CC decreased significantly with age. Furthermore, several studies have suggested that inter-hemispheric communication is better in the female brain than in the male brain, which has prompted investigations of possible sexual dimorphism of the CC (Holloway et al., 1993; Shaywitz et al., 1995; Giedd et al., 1997, 1999; Dubb et al., 2003; Ardekani et al., 2013). For example, Shaywitz et al. (1995) found that the language functions are more likely to be lateralized in males while they are represented in both cerebral hemispheres in females. Additionally, Ardekani et al. (2013) reported that for pairs of male and female individuals with equal brain sizes and similar ages, the CC mid-sagittal area is larger in the female. However, it remains unclear whether these sex differences are similar across different ethnicities.

It has been reported that the size and shape of the brain differ across races (Zilles et al., 2001). Furthermore, studies conducted in different populations have noted that the morphology of the CC seems to exhibit population-related variations that may be influenced by environmental, racial, and genetic factors (Suganthy et al., 2003; Woldehawariat et al., 2014). For example, differences in CC measurements were observed among Indian, Turkish and Japanese populations (Karakaş et al., 2011). Until now, there has been no information on CC morphometric measurements in the Arab ethnic group.

Furthermore, autism is a developmental neurobehavioral condition characterized by difficulties in social communication and interaction, along with restricted repetitive behaviors (Lai et al., 2014). It is $4-5$ times more common in males than females. Autism has been classified into three different levels based on the severity of impairment, with level 1 being the mildest level, requiring minimum support, and level 3 being the most severe level, requiring substantial support (American Psychiatric Association, 2013).

The CC has been the most studied structure in patients with autism spectrum disorder (ASD) since it is speculated that poor brain connectivity in autism may be reflected anatomically in this white matter structure which connects the two cerebral hemispheres (Keary et al., 2009; Lefebvre et al., 2015). It has been hypothesized that the size of the CC is reduced in patients with ASD. However, published findings remain inconclusive with regard to a potential correlation between CC size and autism (Kucharsky Hiess et al., 2015; Lefebvre et al., 2015). Therefore, further research is warranted in order to shed light on this hypothesis, especially in the presence of a wide human neuroanatomical diversity.

To the best of our knowledge, no morphometric analysis of the CC has been performed in any Arabic community. This study aims to assess age- and sex-related morphometric measurements of the CC in Middle Eastern Arabs. This will provide normative anatomical reference data to assist in future diagnostic and disease-related investigations in Middle Eastern individuals. Second, it aims to determine whether there are any significant variations between these measurements and previously reported measurements from different ethnicities. Lastly, this study aims to investigate whether there are any significant differences between normal CC measurements and measurements obtained from patients with ASD.

\section{MATERIALS AND METHODS}

\section{Image Selection}

This study was performed using images for Middle Eastern Arabs stored in the magnetic resonance (MR) imaging unit at King Abdullah University Hospital (KAUH) in Jordan. The Arabic ethnicity was confirmed by the last family name of the subject images, as most Middle Eastern Arabs use their arabic tribe name as their last family name. The study was approved by the Institutional Research Board Committees at Jordan University of Science \& Technology and KAUH (IRB \# GM7601). The work described has been carried out in accordance with the code of ethics of the World Medical Association (1964 Helsinki declaration and its later amendments). For this type of study, formal consent is waived.

The MR images were reviewed and analyzed by specialized radiologists and neurosurgeons to identify those with normal findings (e.g., no focal or enhanced brain lesions, a normal ventricular system, no mass or shift in the midline structures, no hemorrhage, and no fluid collection). In total, MR images were collected from 227 subjects and divided into three age groups as follows: children aged $2-10$ years ( $n=57 ; 29$ males, 28 females), younger adults aged $20-45$ years ( $n=100 ; 40$ males, 60 females), and older adults aged $55-80$ years ( $n=70 ; 34$ males, 36 females).

\section{MR Imaging Protocol}

All subjects were scanned using the same 3-Tesla scanning device (Philips, Andover, MA, United States). Sagittal three-dimensional T1-weighted images were obtained for all subjects using the 
following scanning parameters: 160 sections; slice thickness, $1 \mathrm{~mm}$; echo time, $3.73 \mathrm{~ms}$; repetition time, $8.16 \mathrm{~ms}$; flip angle, $8^{\circ}$; acquisition matrix, 240/240; field of view, $59.3 \times 23.4 \mathrm{~cm}$; one excitation; and total scan time, $15 \mathrm{~min}$. Some of the children were sedated for the scanning.

\section{Identification of Midsagittal Sections}

All sagittal images were retrieved using the Picture Archiving and Communication System (PACS) viewer. The image closest to the midsagittal section of the $\mathrm{CC}$ was selected according to the following criteria described by Pettey and Gee (2002): (i) only traces of cortex visible, (ii) clear separation between the tectum and tegmentum of the midbrain by the cerebral aqueduct, and (iii) good visibility of the fourth ventricle and cerebellar vermis. We also employed the following additional criteria for selecting the midsagittal section: (iv) clear distinction of the septum pellucidum and interthalamic connection and $(\mathrm{v})$ visibility of the anterior and posterior commissures (Figure 1A). Furthermore, viewing the coronal and horizontal series of the sections while simultaneously viewing the sagittal section provided in the PACS software was helpful for determining the true midsagittal section (Figure 1B). All tracing measurements for CC were then performed by two specialized doctors who are very welltrained on using the PACS technology and execute similar measurements continuously on their daily routine work. The inter-rater reliability was evaluated for measurements conducted by both raters, and the average value was more than 0.95 .

\section{Anteroposterior Length Measurements}

The anteroposterior length of the CC was measured by tracing a line connecting the most anterior point with the most posterior point of the CC. The length of the CC relative to the total length of the forebrain (length ratio) was also calculated. To calculate the length ratio of the $\mathrm{CC}$, the anteroposterior length of the forebrain was also measured as the distance between the most anterior point in the frontal lobe and the most posterior point in the occipital lobe.

\section{Area Measurements}

The area of the CC was measured using the PACS software. Each area of the CC was traced manually using the region of interest function within PACS. Two manual tracings were made for each case, and the mean value between the two was calculated. All measurements were obtained in a manner which was blind to the sex and age of the subjects.

\section{Ratio of CC Area to Forebrain Volume}

The ratio of the $\mathrm{CC}$ area to the forebrain volume was also calculated. To measure the forebrain volume, a stereological method was used to extract quantitative information about the three-dimensional volume of the forebrain from measurements made on the two-dimensional sagittal-sectional areas of the MR images. In this study, the forebrain was defined as all gray and white matter, excluding the cerebellum, brain stem, and all structures below the diencephalon (Bermudez and Zatorre, 2001). Moreover, this study used Cavalier's principle, which is the most convenient, efficient, and unbiased method of obtaining volume estimations from serial sections (Mayhew and Olsen, 1991). This method includes the following measurement principles: (i) not every section needs to be analyzed - only every fifth or tenth section does; (ii) sections should be equally spaced; (iii) sections that are analyzed should be systematically sampled; and (iv) the first section being analyzed may not be the first section in which the region of interest appears.

There were 160 sagittal MR image sections for each brain. Sections lacking forebrain tissue, primarily the first and last 10 sections, were excluded. The remaining sections were divided into 14 sets, each of which consisted of 10 sections. Here, we uniformly chose to analyze every fourth section from each set. This uniform selection was important for systematic sampling. Each section in the study was 1-mm thick; therefore, each set was $10 \mathrm{~mm}$ in thickness. The forebrain area of the chosen sections, including the gray and white matter and the ventricles, was then measured using the region of interest tool in PACS. The cerebellum and brain stem were excluded using a cut line from the level of the superior colliculus to the mammillary bodies. The volume was then estimated by multiplying the sum of the areas by the distance between the selected sections $(10 \mathrm{~mm})$ according to the following formula: $V=d \Sigma a$, where $V$ is the forebrain volume, $d$ is the set thickness (distance between selected sections), and $a$ is the cross-sectional area.

\section{Thickness Measurements}

The thickness of the CC was measured at three different sites according to the protocol established by Suganthy et al. (2003). First, the thickness of the genu was measured as the distance from the most anterior point of the genu to the most anterior point of the inner concavity of the CC. Second, the thickness of the body was measured as the dorsoventral height at the midpoint of the CC. Finally, the thickness of the splenium was measured as the distance between two points at the dorsal and ventral margins of the splenium at its widest part (Figure 2).

\section{Racial Comparisons}

In order to assess racial variations in CC size, we conducted racial comparisons between our measurements on Middle Eastern Arabs and previously PubMed-reported measurements on other ethnicities. In order to assure unbiased comparisons and to avoid confounding effects, the following inclusion criteria were applied on the selected studies: (i) the study must be performed on midsagittal MR images (studies conducted on brain specimens or used images from different radiological techniques were excluded); (ii) the study must apply similar definitions for the measurements obtained; (iii) the study subjects must be younger adults (aged between 20 and 50 years), as the comparisons were conducted using the younger adult group only; (iv) the comparison must be sex-separated. Based on the aforementioned criteria we were able to retrieve two studies for two different ethnicities from the PubMed database. These studies included Polish (Guz et al., 2019) and Turkish (Karakaş et al., 2011) populations. The compared measurements included the length, thickness (width), and area of CC. Additionally, the anteroposterior length of the forebrain was included in the 

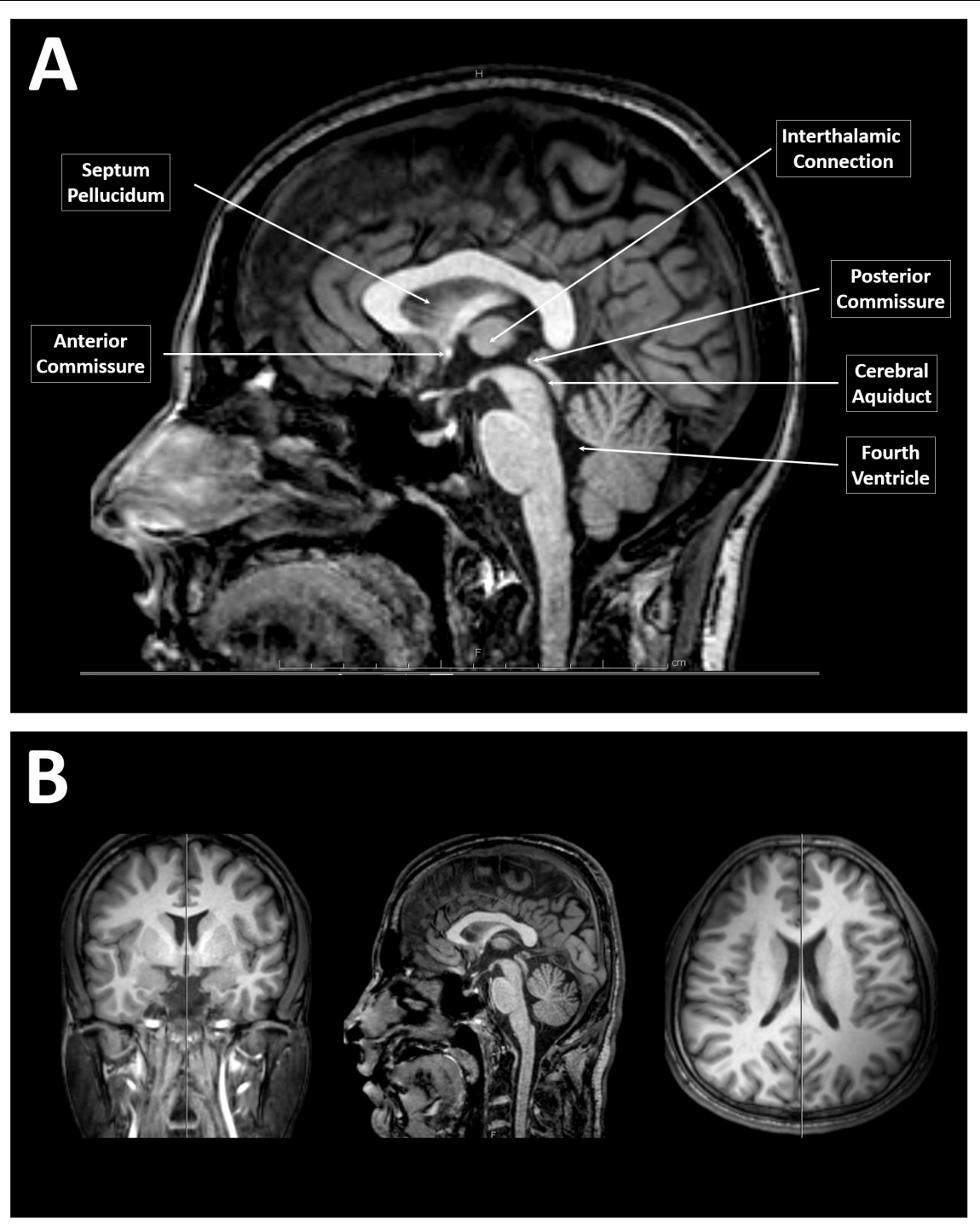

FIGURE 1 | Identification of a midsagittal section from magnetic resonance (MR) images of the brain. (A) A midsagittal MR image of the brain showing the clear separation between the tectum and tegmentum of the midbrain by the cerebral aqueduct, a visible fourth ventricle and cerebellar vermis, clear distinction of the septum pellucidum and interthalamic connection, and visible anterior to posterior commissures. (B) T1-weighted MR images of the brain of a younger adult male that simultaneously showing the coronal, sagittal, and horizontal series of the sections provided by the Picture Archiving and Communication System software.

comparison in order to investigate the variation in the relative length (length ratio) of the CC to the forebrain.

\section{Correlation With Autism}

Mid-sagittal brain MR images were retrieved from KAUH records for 22 male children who were diagnosed with level 2 or 3 autism spectrum disorder. The diagnosis was performed by a clinical expert according to the 5th edition of the Diagnostic and Statistical Manual of mental disorders (DSM-5) published by the American Psychiatric Association, 2013 (American Psychiatric Association, 2013). In summary, the patients had marked to severe deficits in social communication and interaction that included a failure to initiate or respond to social communication, a lack of facial expressions, an inability to understand or use gestures, an inability to make friends and a lack of interest in peers. In addition, the patients displayed repetitive stereotyped motors movements, along with difficulty coping with change and distress when changing their focus or activity.

The boys with ASD were between 5 and 10 years of age at the time of imaging. The CC length, total area, and thickness in the genu, body, and splenium were measured. The findings were then compared with the measurements from the group of neurotypical male children to avoid confounding effects of age and sex.

\section{Statistical Analyses}

All data are presented as the mean \pm standard deviation (SD). For the age analysis, the data were organized into three groups according to whether the images were from 


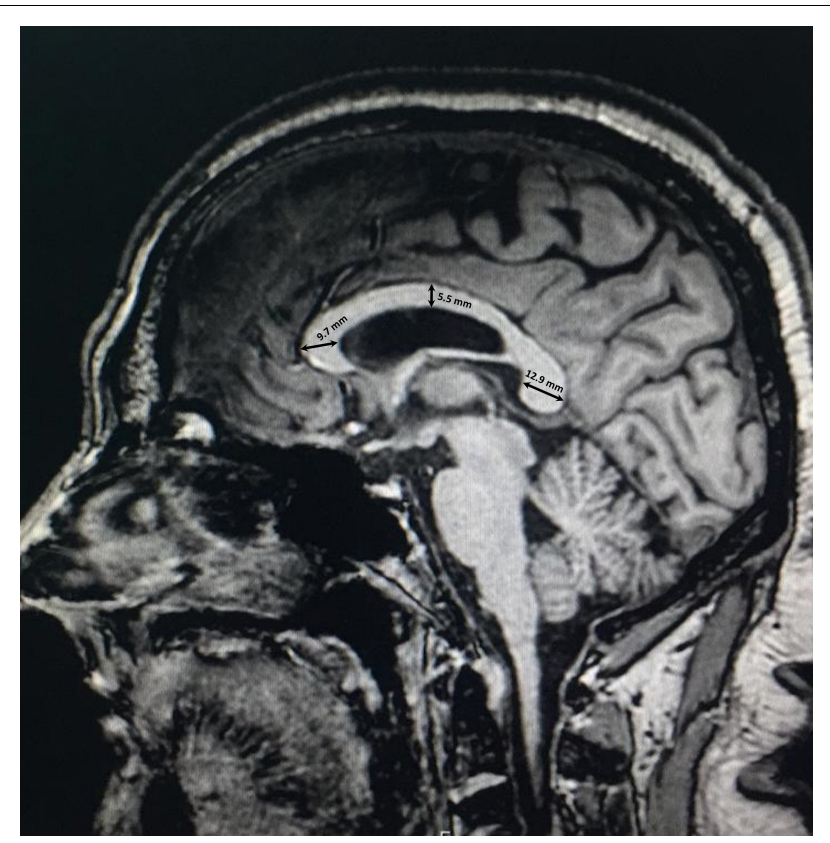

FIGURE 2 | Thickness measurements of the corpus callosum in three regions of the genu, body, and splenium.

children, younger adults, or older adults. First, Levene's test was performed to determine the homogeneity of variance among the three groups. The data were then evaluated with oneway analyses of variance. If a significant difference was found, Fisher's least significant difference post-hoc test was performed to determine the exact statistical differences between the groups. For the sex analysis, the data were divided into two groups according to whether the images were of male or female subjects. Levene's test for equality of variance was performed first, and the data were then evaluated using independentsamples $t$-tests. The one-sample $t$-test was used to perform the racial comparisons. The measurements from either male or female younger adults were used as the test variables, while their corresponding reported mean values from different ethnicities were used as the test values. Lastly, independentsample $t$-test was used to determine the statistical differences between neurotypical boys and boys with ASD as described above. All statistical tests were performed at 5 and $1 \%$ significance levels.

\section{RESULTS}

\section{Age-Related Measurements Anteroposterior (Length) Measurements}

Significantly smaller anteroposterior lengths of the CC $(P=3.3$ $\left.10^{-26}\right)$, forebrain $(P=0.001)$, and the ratio of the $\mathrm{CC}$ length to the forebrain length $\left(P=5.310^{-21}\right)$ were found in children than in younger and older adults (Table 1). No significant differences in the anteroposterior lengths of the CC $(P=0.087)$ and forebrain $(P=0.792)$ were identified between younger and older adults.

\section{Area Measurements}

Significant $\left(P=3.1 \times 10^{-24}\right)$ differences in the total area of the CC were identified among the three age groups (Table 1). The CC area was significantly smaller in children than in younger $\left(P=3.4 \times 10^{-24}\right)$ and older $\left(P=1.1 \times 10^{-5}\right)$ adults and was significantly $\left(P=2.1 \times 10^{-11}\right)$ greater in younger adults than in older adults. A similar trend was found with regard to the ratio of the CC area relative to the forebrain volume (Table 1). The forebrain volume was significantly greater in younger adults than in older adults $\left(P=7.4 \times 10^{-4}\right)$. However, no significant $(P=0.231)$ difference in forebrain volume was observed between children and older adults (Table 1).

\section{Thickness Measurements}

The thickness of the CC in the genu was significantly greater in younger adults than in children $\left(P=3.7 \times 10^{-13}\right)$ and older adults $\left(P=4.0 \times 10^{-10}\right)$, while no significant $(P=0.143)$ differences were found between children and older adults. The thickness of the CC in the body was significantly greater in younger adults than in children $\left(P=4.3 \times 10^{-13}\right)$ and older adults $\left(P=1.9 \times 10^{-10}\right)$, while no significant $(P=0.183)$ differences were found between children and older adults. In the splenium, the CC thickness was significantly larger in younger adults than in children $\left(P=3.4 \times 10^{-14}\right)$ and older adults $(P=0.009)$, and significantly $\left(P=3.7 \times 10^{-7}\right)$ greater in older adults than in children (Table $\mathbf{1}$ ).

\section{Sex-Related Measurements Measurements in Children}

There was no significant $(P=0.485)$ difference in the age between male and female children. The mean ages for male and female children were $5.4 \pm 2.1$ and $5.0 \pm 2.4$ years, respectively. No significant differences in the absolute $(P=0.052)$ or relative $(P=0.533)$ lengths of the CC were found between male and female children. The mean length of the CC was $61.8 \pm 4.4 \mathrm{~mm}$ in males and $59.1 \pm 5.9 \mathrm{~mm}$ in females. However, the forebrain length was significantly $(P=0.003)$ greater in male children than in female children. The mean forebrain lengths in both male and female children were $155.6 \pm 9.7 \mathrm{~mm}$ and $147.2 \pm 10.3 \mathrm{~mm}$, respectively.

The total area of the CC was significantly $(P=0.003)$ greater in male than in female children. The mean area of the CC was $459.5 \pm 85.4 \mathrm{~mm}^{2}$ in males and $395.0 \pm 67.7 \mathrm{~mm}^{2}$ in females. In addition, the forebrain volume was significantly $\left(P=3.0 \times 10^{-7}\right)$ greater in male than in female children. Therefore, no significant difference $(P=0.889)$ in the ratio of the CC area to the forebrain volume was identified between sexes in children.

Although the CC was slightly thicker in male than in female children in the genu $(9.2 \pm 1.6$ vs. $8.5 \pm 1.7 \mathrm{~mm})$, body $(5.3 \pm 1.1$ vs. $4.8 \pm 0.9 \mathrm{~mm})$, and splenium $(13.8 \pm 2.7$ vs. $13.2 \pm 2.1 \mathrm{~mm})$, the differences were not statistically significant ( $P=0.105, P=0.114$, and $P=0.326$; respectively) for all regions.

\section{Measurements in Younger Adults}

There was no significant $(P=0.886)$ difference in the age between male and female younger adults. The mean ages for male and female younger adults were $32.5 \pm 8.8$ and $32.7 \pm 8.8$ years, 
TABLE 1 | Morphometric measurements of the corpus callosum (CC) in three different age groups of Middle Eastern Arabs.

\begin{tabular}{|c|c|c|c|}
\hline Measurement & Children $(n=56)$ & Younger adults $(n=100)$ & Older adults $(n=70)$ \\
\hline \multicolumn{4}{|l|}{ Length measurements } \\
\hline Length of CC (mm) & $60.5 \pm 5.3^{\star *}$ & $68.4 \pm 4.0$ & $69.5 \pm 4.1$ \\
\hline Forebrain length (mm) & $151.5 \pm 10.8^{\star \star}$ & $156.6 \pm 7.6$ & $156.2 \pm 7.5$ \\
\hline Length Ratio & $0.39 \pm 0.03^{\star \star}$ & $0.44 \pm 0.03$ & $0.45 \pm 0.03$ \\
\hline \multicolumn{4}{|l|}{ Area measurements } \\
\hline CC total area $\left(\mathrm{mm}^{2}\right)$ & $427.8 \pm 83.1^{\mathrm{a}}$ & $583.4 \pm 83.8^{b}$ & $493.4 \pm 77.8^{c}$ \\
\hline Forebrain volume $\left(\mathrm{cm}^{3}\right)$ & $1086.2 \pm 126.7^{a, b}$ & $1123.8 \pm 118.4^{b}$ & $1061.0 \pm 109.5^{\mathrm{a}}$ \\
\hline Ratio (CC area/forebrain volume) & $0.39 \pm 0.07^{a}$ & $0.52 \pm 0.07^{b}$ & $0.47 \pm 0.07^{\mathrm{c}}$ \\
\hline \multicolumn{4}{|l|}{ Thickness measurements } \\
\hline Genu thickness (mm) & $8.8 \pm 1.7$ & $10.9 \pm 1.4^{\star \star}$ & $9.2 \pm 1.8$ \\
\hline Body thickness (mm) & $5.0 \pm 1.0$ & $6.2 \pm 0.8^{\star \star}$ & $5.3 \pm 0.8$ \\
\hline Splenium thickness (mm) & $13.5 \pm 2.4^{a \star \star}$ & $16.6 \pm 2.3^{b}$ & $15.6 \pm 2.1^{c}$ \\
\hline
\end{tabular}

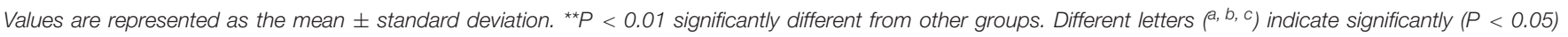
different groups (ANOVA, LSD post-hoc).

respectively. No significant $(P=0.442)$ difference in the length of the CC was observed between male and female younger adults. The mean length of the CC was $68.8 \pm 4.7 \mathrm{~mm}$ in males and $68.1 \pm 3.5 \mathrm{~mm}$ in females. However, the mean length of the forebrain was significantly $\left(P=1.8 \times 10^{-8}\right)$ greater in younger male than in younger female adults $(161.5 \pm 6.7$ vs. $153.3 \pm 6.4 \mathrm{~mm}$ respectively). Therefore, the length ratio of the CC to the forebrain was significantly $\left(P=1.9 \times 10^{-4}\right)$ greater in younger female than in younger male adults.

No significant $(P=0.426)$ difference in the area of the CC was identified between younger male and younger female adults. The mean area of the CC was $592.1 \pm 97.1 \mathrm{~mm}^{2}$ in younger adult males and $577.6 \pm 74.0 \mathrm{~mm}^{2}$ in younger adult females. However, the forebrain volume was significantly $\left(P=3.6 \times 10^{-13}\right)$ greater in males than in females. This was reflected as a significantly $\left(P=7.9 \times 10^{-5}\right)$ greater ratio of the CC area to the forebrain volume in younger female than in younger male adults.

Lastly, no significant difference in thickness was identified between younger male and younger female adults in the genu (10.7 \pm 1.4 vs. $11.0 \pm 1.5 \mathrm{~mm}, P=0.456)$, body $(6.2 \pm 0.8$ vs. $6.1 \pm 0.8 \mathrm{~mm}, P=0.503)$, or splenium $(17.0 \pm 2.5$ vs. $16.3 \pm 2.2$, $P=0.127)$ of the CC.

\section{Measurements in Older Adults}

There was no significant $(P=0.691)$ difference in the age between male and female older adults. The mean ages for male and female older adults were $63.3 \pm 7.9$ and $62.6 \pm 7.0$ years, respectively. The absolute length of the CC was significantly $(P=0.033)$ greater in older male than in older female adults. The mean lengths of the CC in older male and older female adults were $70.6 \pm 3.4 \mathrm{~mm}$ and $68.5 \pm 4.5 \mathrm{~mm}$, respectively. In addition, the mean length of the forebrain was significantly $\left(P=7.0 \times 10^{-9}\right)$ greater in older male than in older female adults $(161.0 \pm 5.0$ vs. $151.7 \pm 6.6 \mathrm{~mm}$, respectively). The length ratio of the $\mathrm{CC}$ to the forebrain length was significantly $(P=0.031)$ greater in older female than in older male adults.

No significant $(P=0.358)$ difference in the area of the CC was found between older male and older female adults. The mean area of the CC was $502.3 \pm 79.8 \mathrm{~mm}^{2}$ in older adult males and $485.0 \pm 76.1 \mathrm{~mm}^{2}$ in older adult females. However, the forebrain volume was significantly $\left(P=2.7 \times 10^{-11}\right)$ greater in males than in females. This was reflected as a significantly $(P=0.003)$ greater ratio of the $\mathrm{CC}$ area to forebrain volume in older female than in older male adults.

Finally, no significant difference in thickness was observed between older male and older female adults in the genu $(9.3 \pm 1.7$ vs. $9.2 \pm 1.8 \mathrm{~mm}, P=0.702$ ), body ( $5.4 \pm 0.8$ vs. $5.2 \pm 0.8 \mathrm{~mm}$, $P=0.376)$, or splenium $(15.8 \pm 2.6$ vs. $15.5 \pm 1.6 \mathrm{~mm}$, $P=0.642)$ of the CC.

\section{Racial Comparisons}

Both male and female racial comparisons showed similar trends in CC variation among different ethnicities (Tables 2, 3). No differences were found in the CC length $(P=0.403$ for males and $P=0.642$ for females $)$ or area $(P=0.057$ for males and $P=0.061$ for females) between Polish and Arab populations. However, the CC was significantly longer $\left(P=1.6 \times 10^{-6}\right.$ for males and $P=1.9 \times 10^{-9}$ for females) in Turkish people than in Arabs, despite the fact that the forebrain was significantly shorter $\left(P=2.6 \times 10^{-10}\right.$ for males and $P=3.1 \times 10^{-4}$ for females) in the Turkish people. The shorter forebrain in the Turkish people resulted in significantly $\left(P=7.8 \times 10^{-17}\right.$ for males and $P=3.4 \times 10^{-17}$ for females) greater length ratio of CC to forebrain in the Turkish people compared to Arabs.

Furthermore, the CC was significantly thicker $\left(P=1.2 \times 10^{-5}\right.$ for males and $P=3.0 \times 10^{-20}$ for females) in Turkish people than in Arabs, except in the splenium region. The CC was significantly ( $P=7.1 \times 10^{-16}$ for male and $P=3.5 \times 10^{-19}$ for females $)$ thicker in the region of the splenium in Middle Eastern Arabs than in the Turkish population (Tables 2, 3).

\section{Autism-Related Measurements}

No significant $(P=0.141)$ difference in the length of the CC was found between neurotypical boys and boys with ASD. However, the area of CC was significantly $(P=0.002)$ smaller in boys with ASD compared to neurotypical boys (Table 4). Similarly, the CC 
TABLE 2 | Racial comparison of the morphometric measurements of the corpus callosum in young adult males between Middle Eastern Arab and other ethnic backgrounds.

\begin{tabular}{|c|c|c|c|c|c|c|c|}
\hline Ethnicity & $\begin{array}{l}\text { Number of } \\
\text { individuals }\end{array}$ & $\begin{array}{l}\text { Forebrain } \\
\text { length (mm) }\end{array}$ & $\begin{array}{l}\text { CC length } \\
(\mathrm{mm})\end{array}$ & $\begin{array}{c}\text { Genu } \\
\text { thickness } \\
\text { (mm) }\end{array}$ & $\begin{array}{c}\text { Body } \\
\text { thickness } \\
(\mathrm{mm})\end{array}$ & $\begin{array}{c}\text { Splenium } \\
\text { width (mm) }\end{array}$ & $\begin{array}{c}\text { Total CC area } \\
\left(\mathrm{mm}^{2}\right)\end{array}$ \\
\hline Middle Eastern Arabs & 40 & $161.5 \pm 6.7$ & $68.8 \pm 4.7$ & $10.7 \pm 1.4$ & $6.2 \pm 0.8$ & $17.0 \pm 2.5$ & $592.1 \pm 97.1$ \\
\hline Polish (Guz et al., 2019) & 101 & $159.0 \pm 7.5$ & $69.4 \pm 5.2$ & - & - & - & $622.2 \pm 86.5$ \\
\hline Turkish (Karakaş et al., 2011) & 23 & $152.5^{\downarrow \downarrow} \pm 5.4$ & $73.0^{\uparrow \uparrow} \pm 5.3$ & $13.2^{\uparrow \uparrow} \pm 2.4$ & $6.9^{\uparrow \uparrow} \pm 2.1$ & $11.9^{\downarrow \downarrow} \pm 1.9$ & - \\
\hline
\end{tabular}

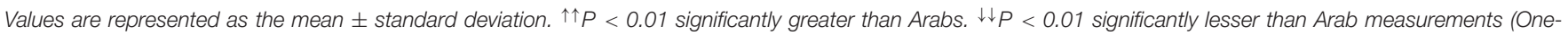
sample t-test).

TABLE 3 | Racial comparisons of morphometric measurements of the corpus callosum in young adult females between Middle Eastern Arab and other ethnic backgrounds.

\begin{tabular}{|c|c|c|c|c|c|c|c|}
\hline Ethnicity & $\begin{array}{l}\text { Number of } \\
\text { individuals }\end{array}$ & $\begin{array}{c}\text { Forebrain } \\
\text { length }(\mathrm{mm})\end{array}$ & $\begin{array}{c}\text { CC length } \\
(\mathrm{mm})\end{array}$ & $\begin{array}{c}\text { Genu width } \\
\text { (mm) }\end{array}$ & $\begin{array}{l}\text { Body width } \\
\text { (mm) }\end{array}$ & $\begin{array}{c}\text { Splenium } \\
\text { width (mm) }\end{array}$ & $\begin{array}{c}\text { Total CC area } \\
\left(\mathrm{mm}^{2}\right)\end{array}$ \\
\hline Middle Eastern Arab & 60 & $153.3 \pm 6.4$ & $68.1 \pm 3.5$ & $11.0 \pm 1.5$ & $6.1 \pm 0.8$ & $16.3 \pm 2.2$ & $577.6 \pm 74.0$ \\
\hline Polish (Guz et al., 2019) & 101 & $151.5 \pm 6.8$ & $68.3 \pm 5.0$ & - & - & - & $595.8 \pm 96.9$ \\
\hline Turkish (Karakaş et al., 2011) & 29 & $150.1^{\downarrow \downarrow} \pm 5.0$ & $71.3^{\uparrow \uparrow} \pm 3.7$ & $13.3^{\uparrow \uparrow} \pm 2.1$ & $7.6 \uparrow \uparrow \pm 1.1$ & $12.5^{\downarrow \downarrow} \pm 1.4$ & - \\
\hline
\end{tabular}

Values are represented as the mean \pm standard deviation. ${ }^{\uparrow} P P<0.01$ significantly greater than Arabs. $\downarrow \downarrow P<0.01$ significantly lesser than Arab measurements $($ Onesample t-test).

was significantly thinner in the genu $(P=0.004)$, body $(P=0.001)$, and splenium $\left(P=2.2 \times 10^{-8}\right)$ of boys with ASD compared to neurotypical boys (Figure 3 and Table 4 ).

\section{DISCUSSION}

This is the first morphometric study of the CC in the Middle Eastern Arab population, and, as such, it provides a reference for future comparisons with other populations, as well as for investigations of forensic and disease-related changes in the CC in Middle Eastern individuals. Several previous studies in the literature have described changes in the morphology of the CC in adults (Going and Dixson, 1990; Holloway et al., 1993; Pettey and Gee, 2002; Dubb et al., 2003; Suganthy et al., 2003) or children (Giedd et al., 1997; Tanaka-Arakawa et al., 2015), while few only have reported these changes across lifespan (Ardekani et al., 2013; Guz et al., 2019). This study uniquely analyzed data collected from Middle Eastern Arab individuals in three age groups, i.e., children, younger adults, and older adults. Our results show that the size of the CC increases significantly from childhood to adulthood and undergoes atrophic changes in thickness rather than length in older adults. Moreover, our finding of sex differences in the area of the CC in children suggests that sexual dimorphism is more likely to be determined by genetic factors than by hormonal or environmental factors. Prominent racial differences were also revealed by the morphometric measurements of the CC. In addition, this study demonstrated that children with autism have distinct changes in CC measurements. The morphometric analyses showed significant reductions in the area and thickness of CC in children with ASD compared to their neurotypical peers.

One source of confusion in morphometric analyses of the CC that may lead to inconsistent results and interpretations
TABLE 4 | Morphometric measurements of the corpus callosum (CC) between neurotypical boys and boys with autism spectrum disorder (ASD).

\begin{tabular}{lcc}
\hline Measurement & Neurotypical $(\boldsymbol{n}=\mathbf{2 8})$ & ASD $(\boldsymbol{n}=\mathbf{2 1})$ \\
\hline Age $(\mathrm{y})$ & $5.4 \pm 2.1$ & $7.6 \pm 1.9$ \\
CC length $(\mathrm{mm})$ & $61.5 \pm 4.0$ & $63.9 \pm 6.3$ \\
CC total area $\left(\mathrm{mm}^{2}\right)$ & $455.3 \pm 83.8$ & $381.9 \pm 80.2^{\text {** }}$ \\
CC thickness (mm) & & \\
Genu & $9.0 \pm 1.5$ & $7.8 \pm 1.3^{\text {** }}$ \\
Body & $5.2 \pm 1.1$ & $4.3 \pm 1.0^{\text {** }}$ \\
Splenium & $13.8 \pm 2.7$ & $9.2 \pm 2.1^{\text {** }}$ \\
\hline
\end{tabular}

Values are represented as the mean \pm standard deviation $(S D)$. ${ }^{* *} P<0.01$ compared to neurotypical boys in the same row (independent t-tests).

is the process of normalization (standardization) of CC measurements to brain size (Bermudez and Zatorre, 2001). Different normalization variables have been suggested as indices of brain size, particularly in studies of sexual dimorphism, such as the forebrain volume, brain weight, cranial capacity, and midsagittal intracranial area (Holloway et al., 1993; Bermudez and Zatorre, 2001; Sullivan et al., 2001). To avoid any such confusion in this study, we considered both the absolute and standardized values for the CC. The standardization method used in this study was the CC value relative to the forebrain volume. The forebrain volume was selected as our standardization variable because of the presence of significant linear and quadratic relationships between absolute CC measurements and forebrain volume, indicating a relatively homogenous enlargement of the CC with increased brain size (Jäncke et al., 1997).

The anteroposterior lengths of the CC and forebrain were significantly shorter in children than in younger and older adults. This finding was expected given that growth and maturation of the CC and forebrain continue until one's mid-twenties 

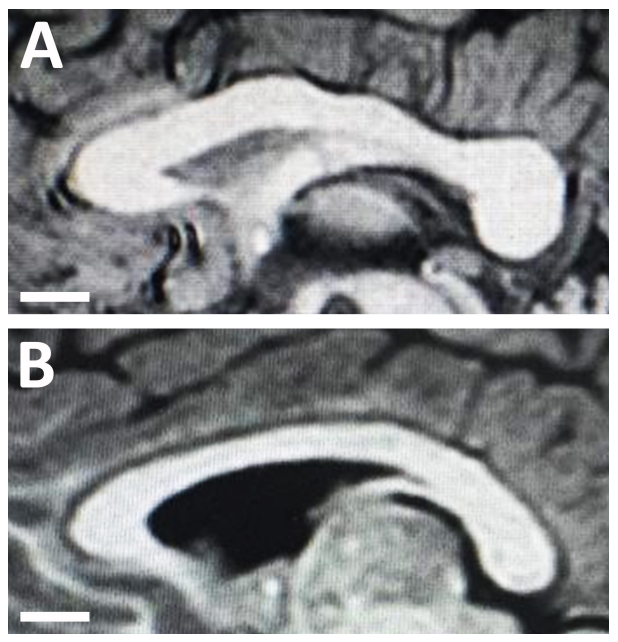

FIGURE 3 | Midsagittal magnetic resonance images of the corpus callosum in (A) an 8-year-old neurotypical male child; and (B) an 8-year-old male child with autism spectrum disorder (ASD). Note the structural changes with autism disorder as the corpus callosum of the child with ASD appears much thinner compared to the callosum of the neurotypical child.
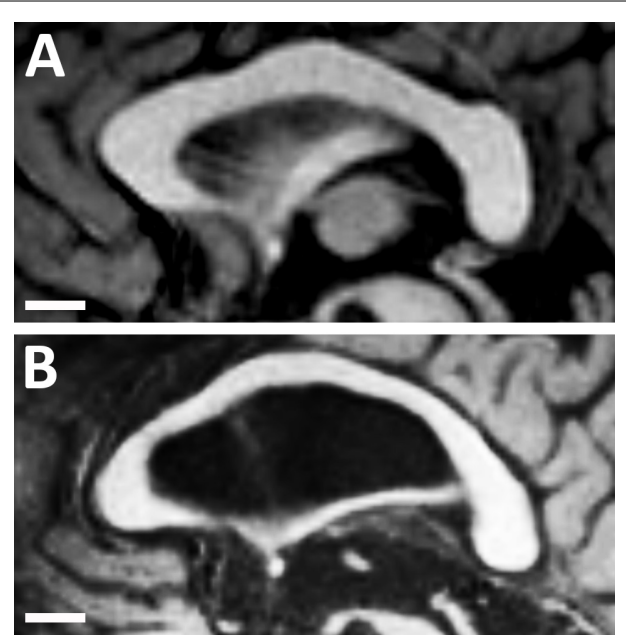

FIGURE 4 | Midsagittal magnetic resonance images of the corpus callosum and lateral ventricle in (A) a healthy younger adult (aged 30 years); and (B) a healthy older adult (aged 68 years). Note the structural changes with age as the lateral ventricle becomes much larger and the corpus callosum becomes much thinner. However, the length of the corpus callosum remains approximately constant.

(Pujol et al., 1993). However, no difference in the length of the CC was found between younger and older adults. This finding contrasted with those of previous studies investigating other populations. For example, Suganthy et al. (2003) reported an increase in the length of the CC with age in an Indian population from the Vellore region, while other studies reported a decrease in CC length with age in the North American (US) population (Byne et al., 1988; Hopper et al., 1994). However, the relative length of the CC to the forebrain length was significantly larger in older adults than in younger adults in our study. This increase in the relative length with age may be explained by the expansion of the lateral ventricles which is associated with generalized brain atrophy in older adults. This expansion of the lateral ventricles could induce structural changes in the CC that may lead to a reduction in its thickness while maintaining its length, thereby preventing length reductions from occurring with age (Figure 4; Sullivan et al., 2002).

In our study, the area and thickness of the CC increased significantly from childhood to adulthood. Comparable results were reported in a previous study that investigated 114 Japanese subjects, including infants, children, adolescents, and adults (Tanaka-Arakawa et al., 2015). Barkovich and Kjos (1988) noted that the CC of newborns appears thin and flat and that the genu undergoes a large increase in thickness at 2 months of age and in the splenium between 4 and 6 months of age. It has been reported that the CC continues to develop together with the cortical brain areas after birth, specifically during the "growth spurt" period of the human cerebral cortex at $\sim 2$ years of age (Matsuzawa et al., 2001; Tanaka-Arakawa et al., 2015). Furthermore, measurements of the total area of the CC in the younger adults in this study were comparable with those of previous MR imaging studies that included different populations (Clarke et al., 1989; Raine et al., 1990; Lacerda et al., 2005). However, a reduction in the area of the CC in older adults was observed when compared with younger adults. This reduction was expected given the brain atrophic changes that occur in older adults. As the brain ages, its sulci and ventricles become larger and the size of the CC decreases owing to the loss of cortical neurons (Going and Dixson, 1990). In addition, there is an age-related reduction in myelination, which would be expected to affect this heavily myelinated structure (Driesen and Raz, 1995).

In children, we found that the area of the CC was significantly larger in males than in females. To our knowledge, this is the first study to report a sex-related difference in CC size in children. This finding may support a previous suggestion that genetic factors play a more important role than environmental or hormonal factors in determining the size of the CC (Woldehawariat et al., 2014). Moreover, the forebrain volume was significantly larger in male than in female children, as is widely known to be the case (Giedd et al., 1997, 1999). The forebrain volume in this study was about $15 \%$ greater in males than in females. The proportional enlargement in both the CC and forebrain in male children resulted in no sex difference in the ratio of the CC area to forebrain volume.

No significant differences in the areas of the CC were identified between younger or older male and female adults. However, the forebrain volume was significantly larger in male subjects in both groups. The smaller forebrain volume in female subjects resulted in a significantly greater ratio of CC area to forebrain volume in younger and older female adults. Comparable findings have been reported in different populations (Jäncke et al., 1997; Tanaka-Arakawa et al., 2015).

With regard to racial comparisons, this study supports the concept that CC dimensions may vary across different ethnic and racial populations. This is comparable to what was reported by Nouri Hosseini et al. (2018), who highlighted that ethnicity 
may influence CC dimensions. However, it is very difficult to draw a solid conclusion regarding the racial variations in CC from our comparison study due to the presence of several limitations. First, the limited number of compared studies as we were able to retrieve only two studies that accomplished similar methodological criteria. Second, the lack of statistical power of the comparisons due to the small number of studied individuals from each population. Third, the unavailability of the raw data from the published studies. The presence of the raw data allows the performance of more comprehensive and more powerful statistical tests which can lead to more rigorous findings and conclusions. Finally, the inability to compare the standardized values of CC size according to the brain volume, as the observed variations might be related to differences in the brain volume rather than to differences in the CC itself.

Future studies are needed to determine the CC measurements across different ethnicities. This will have a greater clinical value, both in stereotaxic surgeries and for identifying abnormalities in these ethnicities. Therefore, we believe it is very important to have an international archive that provides reference data values, not only for CC, but for all brain measurements across different racial groups worldwide.

The presence of a correlation between the size of the CC and autism, and whether this correlation, if present, is causative or associative, remains unknown. Several studies reported no differences in CC size in patients with ASD compared to their neurotypical counterparts (Rice et al., 2005; Kucharsky Hiess et al., 2015; Lefebvre et al., 2015). In contrast, a growing body of literature supports the reduction in CC size in autism as a neuroanatomical sign of poor connectivity and reduced interhemispheric communication, leading to the impaired social and cognitive skills seen in autism (Frazier and Hardan, 2009; Keary et al., 2009; Casanova et al., 2011; Frazier et al., 2012; Prigge et al., 2013). In fact, more recent studies have emphasized that partial agenesis and hypoplasia of the CC could be a causative factor for autism (Wegiel et al., 2017; Patra et al., 2019). Regarding animal experiments on autism, the BTBR and BALB mice are the most widely accepted models since they provide the same core behavioral characteristics as autism (Varghese et al., 2017). Interestingly, the most prominent neuroanatomical feature in these animals is either the absence of CC, or the presence of a CC which is significantly reduced in size (Wahlsten et al., 2003; Fairless et al., 2008).

The exact nature of reduction in the CC size in patients with ASD remains poorly understood. However, it could be attributed to a smaller fiber size, decreased number of white fibers, and/or reduced myelination. A post-mortem study on human brains revealed a decrease in the number of large axons combined with an excessive increase in the number of thin axons within the white matter of patients with ASD (Zikopoulos and Barbas, 2010). In a recent study, Dimond et al. (2019) found a reduced fiber density in the corpus callosum of patients with ASD, suggesting that this reduced density may potentially reflect a decrease in the number of axons. In addition, they found the reduced fiber density in the region of the splenium to be associated with greater social impairment. With regard to myelination, there is a mounting evidence from the literature suggesting that autism is associated with hypomyelination and oligodendrocyte dysfunction (Wei et al., 2016; Kamata et al., 2017; Shen et al., 2018; Graciarena et al., 2019). A genomic-wide transcriptional study demonstrated defects in myelin stability and functionality genes in autism (Richetto et al., 2017). In addition, hypomyelination has been detected in different animal models of autism, including BTBR mice (Wei et al., 2016), mice exposed to valproic acid prenatally (Graciarena et al., 2019), and rats who had been exposed to both fetal inflammation and postnatal hypoxia (van Tilborg et al., 2018). Nevertheless, further studies are required to determine the exact nature of the reduction of CC size in patients with ASD and whether this reduction has a causative or associative relation to autism.

\section{CONCLUSION}

This study shows that CC measurements vary according to age and sex in the Middle Eastern Arab population. With regard to age, the length of the CC was significantly smaller in children compared to both younger and older adults. However, no difference in CC length was observed between younger and older adults, and this finding may be attributed to the ventricular expansion which pressures the CC from inside, allowing it to maintain its length in older adults. In addition, the CC increased significantly in area and thickness from childhood to adulthood. However, significant reductions in these parameters were observed in older adults compared with younger adults, most likely owing to the physiological atrophic changes that occur during aging.

With regard to sexual dimorphism, the sizes of the CC and forebrain were generally larger in male than in female children. This indicates that heritable genetic factors are likely involved in determining the sizes of the CC and forebrain. No sex-related differences in CC area or thickness were observed in younger or older adults. However, the ratio of the CC area to the forebrain volume was generally greater in younger and older female adults due to their smaller forebrains.

Furthermore, this study supports the presence of racial variations in CC measurements. It also reveals that children with autism usually have a smaller CC compared to neurotypical children of the same ethnicity. Further investigations are warranted to determine the exact nature of the reduction in CC size within children with ASD.

\section{DATA AVAILABILITY STATEMENT}

The datasets generated for this study are available on request to the corresponding author.

\section{ETHICS STATEMENT}

The studies involving human participants were reviewed and approved by the Institutional Review Board (IRB) Committee at Jordan University of Science and Technology. Written 
informed consent for participation was not required for this study in accordance with the national legislation and the institutional requirements.

\section{AUTHOR CONTRIBUTIONS}

MZA and MMA contributed to conception and design of the study. HA and SA collected the data and organized the database. MZA performed the statistical analysis. AM revised the study critically for important intellectual content. MZA, MMA, and HA wrote the sections of the manuscript. All authors

\section{REFERENCES}

American Psychiatric Association (2013). Diagnostic and Statistical Manual of Mental Disorders, 5th Edn. Arlington, VA: American Psychiatric Association.

Ardekani, B. A., Figarsky, K., and Sidtis, J. J. (2013). Sexual dimorphism in the human corpus callosum: an MRI study using the OASIS brain database. Cereb. Cortex 23, 2514-2520. doi: 10.1093/cercor/bhs 253

Barkovich, A. J., and Kjos, B. O. (1988). Normal postnatal development of the corpus callosum as demonstrated by MR imaging. AJNR Am. J. Neuroradiol. 9, 487-491.

Bermudez, P., and Zatorre, R. (2001). Sexual dimorphism in the corpus callosum: methodological considerations in MRI morphometry. Neuroimage 13, 11211130. doi: $10.1006 /$ nimg.2001.0772

Byne, W., Bleier, R., and Houston, L. (1988). Variations in human corpus callosum do not predict gender: a study using magnetic resonance imaging. Behav. Neurosci. 102, 222-227. doi: 10.1037/0735-7044.102.2.222

Casanova, M. F., El-Baz, A., Elnakib, A., Switala, A. E., Williams, E. L., Williams, D. L., et al. (2011). Quantitative analysis of the shape of the corpus callosum in patients with autism and comparison individuals. Autism 15, 223-238. doi: $10.1177 / 1362361310386506$

Clarke, S., Kraftsik, R., Van der Loos, H., and Innocenti, G. (1989). Forms and measures of adult and developing human corpus callosum: is there sexual dimorphism? J. Comp. Neurol. 280, 213-230. doi: 10.1002/cne.902800205

Dimond, D., Schuetze, M., Smith, R. E., Dhollander, T., Cho, I., Vinette, S., et al. (2019). Reduced White Matter Fiber Density in Autism Spectrum Disorder. Cereb. Cortex 29, 1778-1788. doi: 10.1093/cercor/bhy348

Driesen, N., and Raz, N. (1995). The influence of sex, age, and handedness on corpus callosum morphology: a meta-analysis. Psychobiology 23, 240-247.

Dubb, A., Gur, R., Avants, B., and Gee, J. (2003). Characterization of sexual dimorphism in the human corpus callosum. Neuroimage 20, 512-519. doi: 10.1016/s1053-8119(03)00313-6

Fairless, A. H., Dow, H. C., Toledo, M. M., Malkus, K. A., Edelmann, M., Li, H., et al. (2008). Low sociability is associated with reduced size of the corpus callosum in the BALB/cJ inbred mouse strain. Brain Res. 1230, 211-217. doi: 10.1016/j.brainres.2008.07.025

Frazier, T. W., and Hardan, A. Y. (2009). A meta-analysis of the corpus callosum in autism. Biol. Psychiatry 66, 935-941. doi: 10.1016/j.biopsych.2009.07.022

Frazier, T. W., Keshavan, M. S., Minshew, N. J., and Hardan, A. Y. (2012). A twoyear longitudinal MRI study of the corpus callosum in autism. J. Autism Dev. Disord. 42, 2312-2322. doi: 10.1007/s10803-012-1478-z

Giedd, J. N., Blumenthal, J., Jeffries, N., Rajapakse, J., Vaituzis, A., Liu, H., et al. (1999). Development of the human corpus callosum during childhood and adolescence: a longitudinal MRI study. Prog. Neuropsychopharmacol. Biol. Psychiatry 23, 571-588. doi: 10.1016/s0278-5846(99)00017-2

Giedd, J. N., Castellanos, F. X., Rajapakse, J. C., Vaituzis, A. C., and Rapoport, J. L. (1997). Sexual dimorphism of the developing human brain. Prog. Neuropsychopharmacol. Biol. Psychiatry 21, 1185-1201.

Going, J., and Dixson, A. (1990). Morphometry of the adult human corpus callosum: lack of sexual dimorphism. J. Anat. 171, 163-167.

Graciarena, M., Seiffe, A., Nait-Oumesmar, B., and Depino, A. M. (2019). Hypomyelination and oligodendroglial alterations in a mouse model of autism spectrum disorder. Front. Cell. Neurosci. 12:517. doi: 10.3389/fncel.2018.00517 contributed to manuscript revision, read and approved the submitted version.

\section{FUNDING}

Funds for the conduction of this study were provided by the Deanship of Research at Jordan University of Science and Technology, Irbid, Jordan (Grant \# 20160212). Publication fees were provided by a grant to MZA from the Office of Research at United Arab Emirates University, Al Ain, UAE (Grant \# G00003289, Fund \# 31M441).

Guz, W., Pazdan, D., Stachyra, S., Świątoń, F., Porêba, P., Bednarz, M., et al. (2019). Analysis of corpus callosum size depending on age and sex. Folia Morphol. 78, 24-32. doi: 10.5603/FM.a2018.0061

Holloway, R. L., Anderson, P. J., Defendini, R., and Harper, C. (1993). Sexual dimorphism of the human corpus callosum from three independent samples: relative size of the corpus callosum. Am. J. Phys. Anthropol. 92, 481-498. doi: 10.1002/ajpa.1330920407

Hopper, K., Patel, S., Cann, T., Wilcox, T., and Schaeffer, J. (1994). The relationship of age, gender, handedness, and sidedness to the size of the corpus callosum. Acad. Radiol. 1, 243-248. doi: 10.1016/s1076-6332(05)80723-8

Huang, H., Zhang, J., Jiang, H., Wakana, S., Poetscher, L., Miller, M. I., et al. (2005). DTI tractography based parcellation of white matter: application to the mid-sagittal morphology of corpus callosum. Neuroimage 26, 195-205.

Jäncke, L., Staiger, J., Schlaug, G., Huang, Y., and Steinmetz, H. (1997). The relationship between corpus callosum size and forebrain volume. Cereb. Cortex 7, 48-56.

Jones, E. G. (1985). "Anatomy, development, and physiology of the corpus callosum," in Epilepsy and the Corpus Callosum, ed. A. G. Reeves (Boston: Springer), 3-20.

Kamata, A., Muramatsu, K., Sawaura, N., Makioka, N., Ogata, T., Kuwashima, M., et al. (2017). Demyelinating neuropathy in a 6-year-old girl with autism spectrum disorder. Pediatr. Int. 59, 951-954. doi: 10.1111/ped.13331

Karakaş, P., Koç, Z., Koç, F., and Gülhal Bozkır, M. (2011). Morphometric MRI evaluation of corpus callosum and ventricles in normal adults. Neurol. Res. 33, 1044-1049.

Keary, C. J., Minshew, N. J., Bansal, R., Goradia, D., Fedorov, S., Keshavan, M. S., et al. (2009). Corpus callosum volume and neurocognition in autism. J. Autism Dev. Disord. 39, 834-841. doi: 10.1007/s10803-009-0689-4

Kucharsky Hiess, R., Alter, R., Sojoudi, S., Ardekani, B. A., Kuzniecky, R., and Pardoe, H. R. (2015). Corpus callosum area and brain volume in autism spectrum disorder: quantitative analysis of structural MRI from the ABIDE database. J Autism Dev Disord 45, 3107-3114. doi: 10.1007/s10803-015-2468-8

Lacerda, A., Brambilla, P., Sassi, R., Nicoletti, M., Mallinger, A., Frank, E., et al. (2005). Anatomical MRI study of corpus callosum in unipolar depression. J. Psychiatr. Res. 39, 347-354.

Lai, M. C., Lombardo, M. V., and Baron-Cohen, S. (2014). Autism. Lancet 383 , 896-910. doi: 10.1016/S0140-6736(13)61539-1

LaMantia, A. S., and Rakic, P. (1990). Axon overproduction and elimination in the corpus callosum of the developing rhesus monkey. J. Neurosci. 10, 2156-2175.

Lefebvre, A., Beggiato, A., Bourgeron, T., and Toro, R. (2015). Neuroanatomical diversity of corpus callosum and brain volume in autism: meta-analysis, analysis of the autism brain imaging data exchange project, and simulation. Biol. Psychiatry 78, 126-134. doi: 10.1016/j.biopsych.2015.02.010

Matsuzawa, J., Matsui, M., Konishi, T., Noguchi, K., Gur, R. C., Bilker, W., et al. (2001). Age-related volumetric changes of brain gray and white matter in healthy infants and children. Cereb. Cortex 11, 335-342.

Mayhew, T., and Olsen, D. (1991). Magnetic resonance imaging (MRI) and modelfree estimates of brain volume determined using the Cavalieri principle. J. Anat. 178, 133-144.

Mooshagian, E. (2008). Anatomy of the corpus callosum reveals its function J. Neurosci. 28, 1535-1536. 
Nouri Hosseini, H., Mohammadi, M. R., Aarabi, M., Mohammadi, N., and Golalipour, M. J. (2018). Ethnicity influences corpus callosum dimensions. Neurol. Res. Int. 2018:8916035. doi: 10.1155/2018/8916035

Patra, S., Naik, S., and Jha, M. (2019). Corpus callosum agenesis: neuroanatomical model of autism spectrum disorder? Indian J. Psychol. Med. 41, 284-286. doi: 10.4103/IJPSYM.IJPSYM_281_18

Pettey, D., and Gee, J. (2002). Sexual dimorphism in the corpus callosum: a characterization of local size variations and a classification driven approach to morphometry. Neuroimage 17, 1504-1511.

Prigge, M. B., Lange, N., Bigler, E. D., Merkley, T. L., Neeley, E. S., Abildskov, T. J., et al. (2013). Corpus Callosum Area in Children and Adults with Autism. Res. Autism Spectr. Disord. 7, 221-234.

Pujol, J., Vendrell, P., Junqué, C., Martí-Vilalta, J., and Capdevila, A. (1993). When does human brain development end? Evidence of corpus callosum growth up to adulthood. Ann. Neurol. 34, 71-75.

Raine, A., Harrison, G., Reynolds, G., Sheard, C., Cooper, J., and Medley, I. (1990). Structural and functional characteristics of the corpus callosum in schizophrenics, psychiatric controls, and normal controls. A magnetic resonance imaging and neuropsychological evaluation. Arch. Gen. Psychiatry 47, 1060-1064.

Rice, S. A., Bigler, E. D., Cleavinger, H. B., Tate, D. F., Sayer, J., McMahon, W., et al. (2005). Macrocephaly, corpus callosum morphology, and autism. J. Child Neurol. 20, 34-41.

Richetto, J., Chesters, R., Cattaneo, A., Labouesse, M. A., Gutierrez, A. M. C., Wood, T. C., et al. (2017). Genome-wide transcriptional profiling and structural magnetic resonance imaging in the maternal immune activation model of neurodevelopmental disorders. Cereb. Cortex 27, 3397-3413. doi: 10.1093/ cercor/bhw320

Sakai, T., Mikami, A., Suzuki, J., Miyabe-Nishiwaki, T., Matsui, M., Tomonaga, M., et al. (2017). Developmental trajectory of the corpus callosum from infancy to the juvenile stage: comparative MRI between chimpanzees and humans. PLoS One 12:e0179624. doi: 10.1371/journal.pone.0179624

Shaywitz, B., Shaywitz, S., Pugh, K., Constable, R., Skudlarski, P., Fulbright, R., et al. (1995). Sex differences in the functional organization of the brain for language. Nature 373, 607-609.

Shen, H. Y., Huang, N., Reemmer, J., and Xiao, L. (2018). Adenosine actions on oligodendroglia and myelination in autism spectrum disorder. Front. Cell Neurosci. 12:482. doi: 10.3389/fncel.2018.00482

Suganthy, J., Raghuram, L., Antonisamy, B., Vettivel, S., Madhavi, C., and Koshi, R. (2003). Gender- and age-related differences in the morphology of the corpus callosum. Clin. Anat. 16, 396-403.

Sullivan, E., Pfefferbaum, A., Adalsteinsson, E., Swan, G. E., and Carmelli, D. (2002). Differential rates of regional brain change in callosal and ventricular size: a 4-year longitudinal MRI study of elderly men. Cereb. Cortex 12, 438-445.

Sullivan, E., Rosenbloom, M., Desmond, J., and Pfefferbaum, A. (2001). Sex differences in corpus callosum size: relationship to age and intracranial size. Neurobiol. Aging 22, 603-611.
Tanaka-Arakawa, M., Matsui, M., Tanaka, C., Uematsu, A., Uda, S., Miura, K., et al. (2015). Developmental changes in the corpus callosum from infancy to early adulthood: a structural magnetic resonance imaging study. PLoS One 10:e0118760. doi: 10.1371/journal.pone.0118760

van Tilborg, E., Achterberg, E. J. M., van Kammen, C. M., van der Toorn, A., Groenendaal, F., Dijkhuizen, R. M., et al. (2018). Combined fetal inflammation and postnatal hypoxia causes myelin deficits and autism-like behavior in a rat model of diffuse white matter injury. Glia 66, 78-93. doi: 10.1002/glia. 23216

Varghese, M., Keshav, N., Jacot-Descombes, S., Warda, T., Wicinski, B., Dickstein, D. L., et al. (2017). Autism spectrum disorder: neuropathology and animal models. Acta Neuropathol. 134, 537-566. doi: 10.1007/s00401-017-1736-4

Wahlsten, D., Metten, P., and Crabbe, J. C. (2003). Survey of 21 inbred mouse strains in two laboratories reveals that BTBR $\mathrm{T} / \mathrm{t} \mathrm{tf} / \mathrm{tf}$ has severely reduced hippocampal commissure and absent corpus callosum. Brain Res. 971, 47-54.

Wegiel, J., Flory, M., Kaczmarski, W., Brown, W. T., Chadman, K., Wisniewski, T., et al. (2017). Partial agenesis and hypoplasia of the corpus callosum in idiopathic autism. J. Neuropathol. Exp. Neurol. 76, 225-237. doi: 10.1093/jnen/nlx003

Wei, H., Ma, Y., Liu, J., Ding, C., Hu, F., and Yu, L. (2016). Proteomic analysis of cortical brain tissue from the BTBR mouse model of autism: Evidence for changes in STOP and myelin-related proteins. Neuroscience 312, 26-34. doi: 10.1016/j.neuroscience.2015.11.003

Weis, S., Kimbacher, M., Wenger, E., and Neuhold, A. (1993). Morphometric analysis of the corpus callosum using MR: correlation of measurements with aging in healthy individuals. AJNR Am. J. Neuroradiol. 14, 637-645.

Woldehawariat, G., Martinez, P. E., Hauser, P., Hoover, D. M., Drevets, W. W., and McMahon, F. J. (2014). Corpus callosum size is highly heritable in humans, and may reflect distinct genetic influences on ventral and rostral regions. PLoS One 9:e99980. doi: 10.1371/journal.pone.0099980

Zikopoulos, B., and Barbas, H. (2010). Changes in prefrontal axons may disrupt the network in autism. J. Neurosci. 30, 14595-14609. doi: 10.1523/JNEUROSCI. 2257- 10.2010

Zilles, K., Kwawashima, R., Dabrinhaus, A., Fukuda, H., and Schormann, T. (2001). Hemispheric shape of European and Japanese brains. Neuroimage 13, 262-271.

Conflict of Interest: The authors declare that the research was conducted in the absence of any commercial or financial relationships that could be construed as a potential conflict of interest.

Copyright (C) 2020 Allouh, Al Barbarawi, Ali, Mustafa and Alomari. This is an open-access article distributed under the terms of the Creative Commons Attribution License (CC BY). The use, distribution or reproduction in other forums is permitted, provided the original author(s) and the copyright owner(s) are credited and that the original publication in this journal is cited, in accordance with accepted academic practice. No use, distribution or reproduction is permitted which does not comply with these terms. 